\title{
Hall effect in organic layered conductors
}

\author{
R.A.Hasan ${ }^{1}$, M.V.Kartsovnik², V.G.Peschansky ${ }^{1,3}$ \\ 1 V.N.Karazin Kharkiv national university \\ Svoboda Sqr., 4, Kharkiv 61077, Ukraine \\ 2 Walther-Meissner Institute, \\ Walther-Meissner Str. 8, \\ D-85748 Garching, Germany \\ ${ }^{3}$ B.I.Verkin Institute for Low Temperature \\ Physic and Engineering \\ Lenin Ave., 47, Kharkiv 61103, Ukraine \\ Received July 18, 2005, in final form February 13, 2006
}

The Hall effect in organic layered conductors with a multisheeted Fermi surfaces was considered. It is shown that the experimental study of Hall effect and magnetoresistance anisotropy at different orientations of current and a quantizing magnetic field relative to the layers makes it possible to determine the contribution of various charge carriers groups to the conductivity, and to find out the character of Fermi surface anisotropy in the plane of layers

Key words: Hall effect, organic layered conductors, multisheeted Fermi surface

PACS: $72.15 . \mathrm{Gd}, 75.70 . \mathrm{Cn}$

Galvanomagnetic phenomena in degenerated conductors placed in a strong magnetic field $\mathbf{B}$, when cyclotron frequency of electrons $\omega_{\mathrm{B}}$ considerably exceeds their collision frequency $1 / \tau$, are very sensitive to the form of the electron energy spectrum $[1,2]$. Experimental studies of the magnetoresistance anisotropy were successfully utilized as a simple spectroscopic method for the reconstruction of the Fermi surface (FS) topology. Investigations of the Hall effect provide important information about the charge carriers. In conductors with reduced dimensionality, the Hall effect manifests itself in the manner essentially different from the conventional metals. For example, in two-dimensional structures, the Hall field orthogonal to the current density on the magnitude of quantizing magnetic field has got a step like form [3,4]. At the same time, in quasi-isotropic conductors, at $\omega_{\mathrm{B}} \tau \ll 1$, the orbital quantization of electrons in a magnetic field being taken into account does not essentially effect the magnitude of the Hall field.

In uncompensated metals with closed FS $\epsilon(\mathbf{p})=\epsilon_{\mathrm{F}}$, the Hall field orthogonal to the current $\mathbf{J}$, in collisionless limit $(\tau \rightarrow \infty)$, has the following asymptotic form

$$
\mathbf{E}_{\text {Hall }}=\frac{[\mathbf{J} \times \mathbf{B}]}{N e c} .
$$

The corrections to the Hall field, arising from the quantization of electron energy in the magnetic field, occur only in the following approximations in the small parameter $\gamma=1 / \omega_{\mathrm{B}} \tau \gg 1$.

Here $e$ is the electron charge, $c$ is the velocity of light in vacuum, $N=N_{1}-N_{2}, N_{1}$ is the number of electrons in a unit volume, and $N_{2}$ is the number of holes, whose velocity vector $\mathbf{v}=\partial \epsilon(\mathbf{p}) / \partial \mathbf{p}$ is directed within the closed concavity of isoenergetic surface. In stationary electric $\mathbf{E}$ and magnetic fields, the drift velocity of charge carriers in a plane, orthogonal to the magnetic field, is identical to all conduction electrons

$$
\mathbf{u}=c \frac{[\mathbf{E} \times \mathbf{B}]}{B^{2}} .
$$

Since the directions of motion along the closed orbits of charge carriers $\epsilon(\mathbf{p})=$ const, $p_{\mathrm{B}}=$ $\mathbf{p B} / B=$ const in the electronic and hole state are different, the current density in collisionless 
limit takes the form

$$
\mathbf{J}=N_{1} e \mathbf{u}-N_{2} e \mathbf{u}
$$

which in view of the relation (2) gives an asymptotical expression (1) for the Hall field.

This result has been obtained by I.M. Lifshits [5] in one of the first theoretical papers devoted to the analysis of galvanomagnetic phenomena in metals with arbitrary dispersion law of charge carriers in the quantizing magnetic field.

The asymptote of Hall field (1) at $\gamma \ll 1$ is also valid in the case of the FS in the form of corrugated cylinder even in the presence of open sections of this surface in a magnetic field, orthogonal to the axis of the cylinder [6]. This is connected with the fact that the drift of charge carriers along the open orbits in the plane, orthogonal to the magnetic field, turns out to be compensated in the expression for the current density in collisionless limit. In the case of more complicated open FS, for example a space or plane nets of cylinders, the asymptote of Hall field cannot be described by the general expression (1) and essentially depends on the orientation of the magnetic field. The reason is that in this case, depending on the magnetic field direction, the same state of charge carriers can be either an electron or a hole [1].

FS of the organic conductors with layered structure is open and weakly corrugated along the momentum projection $p_{z}=\mathbf{p n}$ on the normal to layers $\mathbf{n}$. Quasi-two-dimensional character of electron energy spectrum of layered conductors essentially distinguishes them from the perfect two- dimensional structures, as well as from quasi-isotropic metals.

Let us consider Galvanomagnetic phenomena in organic layered conductors with an arbitrary dispersion law of charge carriers

$$
\begin{aligned}
\epsilon(\mathbf{p}) & =\sum_{n=0}^{\infty} \epsilon_{n}\left(p_{x}, p_{y}\right) \cos \left(\frac{a n p_{z}}{\hbar}+\alpha_{n}\left(p_{x}, p_{y}\right)\right) ; \\
\epsilon_{n}\left(-p_{x},-p_{y}\right) & =\epsilon_{n}\left(p_{x}, p_{y}\right), \quad \alpha_{n}\left(-p_{x},-p_{y}=-\alpha_{n}\left(p_{x}, p_{y}\right) .\right.
\end{aligned}
$$

It is assumed that the functions $\epsilon_{n}\left(p_{x}, p_{y}\right)$ and $\alpha_{n}\left(p_{x}, p_{y}\right)$ are arbitrary, and $\epsilon_{n}\left(p_{x}, p_{y}\right)$ is rapidly decreasing with the number $n$, so that the maximum value of function $\left\{\epsilon(\mathbf{p})-\epsilon_{0}\left(p_{x}, p_{y}\right)\right\}$, which is equal to $\eta \epsilon_{\mathrm{F}}$ on the Fermi surface, is much smaller than the Fermi energy $\epsilon_{\mathrm{F}}$

$$
\max \left\{\epsilon(\mathbf{p})-\epsilon_{0}\left(p_{x}, p_{y}\right)\right\}=\eta \epsilon_{\mathrm{F}} \ll \epsilon_{\mathrm{F}} .
$$

Here $a$ is the spacing between adjacent layers, $\hbar$ is Planck's constant.

Experimental observation of Shubnikov-de Haas oscillations (SdH) in the magnetoresistance of practically all layered conductors of an organic origin over a wide interval of the angles $\theta$ between the vectors $\mathbf{B}$ and $\mathbf{n}$ proves that at least one cavity of their FS is a slightly corrugated cylinder. Velocity distributions of charge carriers in the plane of layers for such FS have no essential anisotropy, and for the sake of convenience of evaluations we suppose that the function $\epsilon_{0}\left(p_{x}, p_{y}\right)$ is isotropic and of the form

$$
\epsilon_{0}\left(p_{x}, p_{y}\right)=\frac{p_{x}^{2}+p_{y}^{2}}{2 m}
$$

moreover, all the rest functions $\epsilon_{n}\left(p_{x}, p_{y}\right)$ with $n \gg 1$ are equal to stationary values.

By making use of the equation of motion of a charge in the magnetic field

$$
\begin{aligned}
& \mathbf{B}=(B \cos \varphi \sin \theta, B \sin \varphi \sin \theta, B \cos \theta), \\
& \frac{\partial p_{x}}{\partial t}=\frac{e B \cos \theta}{c}\left(v_{y}-v_{z} \sin \varphi \tan \theta\right), \\
& \frac{\partial p_{y}}{\partial t}=\frac{e B \cos \theta}{c}\left(v_{z} \cos \varphi \tan \theta-v_{y}\right),
\end{aligned}
$$

it is easy to find the contribution to the components of conductivity tensor $\sigma_{i j}$ from a group of charge carriers whose states belong to a sheet of the FS having the form of a slightly corrugated cylinder

$$
\sigma_{x x}=\frac{\gamma^{2} \sigma_{0}}{1+\gamma^{2}}+\sigma_{z z} \frac{\tan ^{2} \theta}{\left(1+\gamma^{2}\right)^{2}}\left(\cos ^{2} \varphi-\gamma^{2} \sin ^{2} \varphi\right)
$$




$$
\begin{gathered}
\sigma_{y y}=\frac{\gamma^{2} \sigma_{0}}{1+\gamma^{2}}+\sigma_{z z} \frac{\tan ^{2} \theta}{\left(1+\gamma^{2}\right)^{2}}\left(\sin ^{2} \varphi-\gamma^{2} \cos ^{2} \varphi\right) \\
\sigma_{x y}=\frac{\gamma \sigma_{0}}{1+\gamma^{2}}+\sigma_{z z} \frac{\tan ^{2} \theta}{1+\gamma^{2}}\left(\sin \varphi \cos \varphi-\frac{\gamma}{1+\gamma^{2}}\right) \\
\sigma_{y z}=\sigma_{z z} \frac{\tan \theta}{1+\gamma^{2}}(\sin \varphi-\gamma \cos \varphi) \\
\sigma_{z x}=\sigma_{z z} \frac{\tan \theta}{1+\gamma^{2}}(\cos \varphi-\gamma \sin \varphi)
\end{gathered}
$$

where $\gamma=1 / \omega_{\mathrm{B}} \tau, \omega_{\mathrm{B}}=e B \cos \theta / m c$ coincides with the cyclotron frequency of electrons in the magnetic field in the leading approximation in the energy spectrum quasi-two-dimensionality parameter $\eta$.

When the FS of a layered conductor consists only of one cylinder, the Hall field linearly grows with the magnetic field $[6,7]$ and quantum oscillations in the strong magnetic field arise only in the following approximations in parameter $\gamma$, the same as in uncompensated metals.

A considerable group of layered organic conductors have multisheeted FS, and therefore consist of various topological elements such as corrugated cylinders and planes slightly corrugated along $p_{z}$ axis. For example, such is the FS of organic conductors based on tetrathiafulvalene $(\mathrm{BEDT}-\mathrm{TTF})_{2} \mathrm{M}(\mathrm{SCN})_{4}$, where $\mathrm{M}$ is one of the metals $\mathrm{Na}, \mathrm{K}, \mathrm{Tl}$, or $\mathrm{NH}_{3}$.

One can choose the coordinate system so that the plane adjoining the slightly corrugated planes of the FS is parallel to the $p_{y} p_{x}$ plane. At any orientation of a magnetic field there are open sections of corrugated plane $p_{\mathrm{B}}=$ const, and charge carriers drift along the open trajectories, generally, along $\mathrm{x}$ axis with the velocity

$$
\bar{v}_{x}=\frac{c\left(p_{y}(0)-p_{y}(T)\right)}{T e B \cos \theta} .
$$

Time $T$ can be interpreted as the time of an electron motion along the open trajectory in the momentum space for a distance about the period along $p_{y}$ axis or $p_{z}$ axis.

After averaging the equation of motion of charge (8) over a sufficiently long time interval about the order of the mean free time of electron $\tau$, one can obtain the following relation:

$$
\bar{v}_{y}=\bar{v}_{z} \sin \varphi \tan \theta
$$

therefore, at angles $\theta$ noticeably different from $\pi / 2$, the drift of charge carriers along $y$ axis is rather small.

When the current flows along the $x$ axis, the main contribution to the total electric conductivity

$$
\sigma_{i j}=\sigma_{i j}^{(1)}+\sigma_{i j}^{(2)}
$$

is made by charge carriers, whose states belong to the corrugated plane of FS. Here, the matrix $\sigma^{(2)_{i j}}$ takes into account the contribution to electric conductivity tensor from charge carriers whose states belong to the corrugated cylinder, and the components of conductivity tensor $\sigma^{(1)_{i j}}$ are due to the presence of additional group of charge carriers belonging to the FS in the form of slightly corrugated planes. The asymptote of the component in a strong magnetic field is equal to $\sigma_{1}$. And, as follows from relation (14), it has the same order as in the absence of magnetic field.

Keeping in mind the relation (15), it is not hard to show that

$$
\sigma_{y y}^{(1)}=\sigma_{z z}^{(1)} \sin ^{2} \varphi \tan ^{2} \theta+\gamma_{1}^{2} \sigma_{2}
$$

where $\sigma_{2}$ is the magnitude of the same order as $\sigma_{1}$, and $\gamma_{1}=T / 2 \pi \tau$. Hereinafter, we shall not distinguish $\gamma$ and $\gamma_{1}$ in the expression for $\sigma_{i j}$ in a sufficiently high magnetic field.

The non-diagonal component $\sigma_{x y}^{(1)}$ is much smaller than $\sigma_{x y}^{(2)}$. And its asymptote in the high magnetic field

$$
\sigma_{x y}^{(1)}=\frac{2 e^{2}}{(2 \pi \hbar)^{3}} \int_{0}^{2 \pi \hbar \cos \theta / a} \mathrm{~d} p_{\mathrm{H}} \frac{c}{e H \cos ^{2} \theta} \int_{0}^{p_{y}(T)-p_{y}(0)}\left(p_{x}-\bar{p}_{x}\right) \mathrm{d} p_{y}
$$


is determined by the degree of corrugation of the FS plane $p_{x} p_{y}$. Here $\bar{p}_{x}$ is the average value of $p_{x}(t)$ on the electronic orbit, and so the integrand is sign-variable.

There are sufficient grounds to consider that the energy spectrum of charge carriers with a FS in the form of corrugated planes in the above mentioned salts is quasi-one-dimensional $[8,9]$. In this case $\sigma_{2} \ll \sigma_{1}$ and Hall components of the magnetoresistance tensor takes the form

$$
\begin{gathered}
\rho_{x y}=\frac{H \cos \theta}{N e c\left(1+\sigma_{1} / \sigma_{0}\right)}, \quad \rho_{z x}=\frac{H \sin \theta \sin \varphi}{N e c\left(1+\sigma_{1} / \sigma_{0}\right)}, \\
\rho_{y z}=\frac{H \cos \varphi \sin \theta}{N e c}-\frac{H^{2} \sin \varphi \sin 2 \theta}{2(N e c)^{2}} \frac{\sigma_{1}}{\sigma_{1}+\sigma_{0}},
\end{gathered}
$$

where $N$ is the density of carrier group whose states belong to a slightly corrugated cylinder.

The studies of Hall field together with the dissipation components of the magnetoresistance tensor [10]

$$
\rho_{x x}=\frac{1}{\sigma_{1}+\sigma_{0}} ; \quad \rho_{y y}=\frac{1}{\sigma_{0}}+\frac{H^{2} \sigma_{1} \cos ^{2} \theta}{(N e c)^{2}\left(1+\sigma_{1} / \sigma_{0}\right)}
$$

allow us to separately determine the contributions to electric conductivity from various groups of charge carriers.

At rather low temperatures when the distance between quantized Landau levels exceeds the temperature smearing of the Fermi distribution function of charge carriers, the collision frequency of electrons with FS in the form of cylinder exhibits a quantum oscillation

$$
\frac{1}{\tau}=\frac{1}{\tau_{0}}\left(1+\Delta_{\mathrm{osc}}\right)
$$

where $\Delta_{\text {osc }}$ is the quantum correction to the collision frequency of the electrons, oscillating as a function of $1 / H$ [11-13]. At the same time, the charge carriers whose states belong to the corrugated plane do not give any contribution to quantum oscillation effect as their energy distribution is essentially continuous.

Although these charge carriers do not participate in forming SdH oscillations, their presence results in a substantial growth of the amplitude of the Hall field quantum oscillations. In conductors with multi-sheeted FS, the relative amplitude of the Hall field oscillations

$$
\frac{E_{\mathrm{Hall}}^{\mathrm{osc}}}{E_{\mathrm{Hall}}^{\mathrm{mon}}}=\Delta_{\mathrm{osc}} \frac{\sigma_{1}}{\sigma_{1}+\sigma_{0}}
$$

is of the same order of magnitude as the magnitoresistanse oscillations.

In magnetic fields substantially accessible and satisfying the requirement

$$
\eta \ll \gamma \ll 1
$$

it is easy to perform quite informative formulas for the ratio of Hall field and the electric field along the current under the most general assumptions concerning the dispersion law of charge carriers.

In the expansion of the magnetoresistance tensor components ratio $E_{\beta} / E_{\alpha}=\rho_{\beta \alpha} / \rho_{\alpha \alpha}$ in terms of $\gamma$, in this work we will neglect the terms proportional to $\gamma^{2}$ as small corrections. Therefore, we shall keep just the first two summands.

In this approximation, when current flows along the $x$ axis, i.e. $\alpha=x$, the ratio of non-diagonal Hall components of the electroresistance tensor $\rho_{\beta x}$ and $\rho_{x x}$ take the form:

$$
\frac{\rho_{y x}}{\rho_{x x}}=-\frac{\sigma_{x y}}{\sigma_{y y}} ; \quad \frac{\rho_{z x}}{\rho_{x x}}=-\cos \varphi \tan \theta+\frac{\sigma_{x y}}{\sigma_{y y}} .
$$

Besides, the linear growth in the magnetic field $E_{z}$ contains the terms that depend just on the orientation of this magnetic field. 
When the current flow along the Hall field is directed predominantly along the normal to the layers and quadratically grows in the magnetic field as well as the electric field along the current $E_{y}$, until $\sin \varphi \gg \gamma$

$$
\frac{\rho_{z y}}{\rho_{y y}}=-\sin \varphi \tan \theta-\frac{\sigma_{x y}}{\sigma_{1}} \cos \varphi \tan \theta ; \quad \frac{\rho_{x y}}{\rho_{y y}}=\frac{\sigma_{z z}}{\sigma 1} \tan ^{2} \theta \sin \varphi \cos \varphi+\frac{\sigma_{x y}}{\sigma_{1}} .
$$

The magnetoresistance across the layers $\rho_{z z}$, with a sufficient order of accuracy, is equal to $1 / \sigma_{z z}$. Therefore, knowing $\rho_{z z}$, it is possible to determine $\sigma_{1}$ using the component of the expression (26) independent of $B$ for $\rho_{x y} / \rho_{y y}$.

The main contribution to the component of conductivity tensor $\sigma_{x y}=\sigma_{x y}^{(1)}+\sigma_{x y}^{(2)}$ is made by charge carriers, whose states belong to the corrugated cylinder of FS,

$$
\sigma_{x y}^{(2)}=\frac{N e c}{H \cos \theta}
$$

and the density of charge carriers of this group $N=\left(S_{\max }+S_{\min }\right) / 2 a(2 \pi \hbar)^{2}$ can be found from measuring SdH oscillations, whose period is determined by the extremal cross-sections of the cylinder $S_{\max }$ and $S_{\min }$.

If the number $N$ of charge carriers whose states belong to the weakly corrugated cylinder is known, one can determine the contributions of both groups of conduction electrons in the absence of a magnetic field by solving the equations (21) with respect to $\sigma_{0}$ and $\sigma_{1}$. Therefore, having experimentally determined the constant of proportionality $\gamma=\mathrm{Nec} / / \sigma_{0} H \cos \theta$ in the expression (26) for the ratio of the Hall field and the electric field $E_{y}$, it is possible to determine the fraction of participation of electrons on the corrugated plane of FS in the Hall component of the conductivity tensor $\sigma_{x y}$. In the case of quasi-one-dimensional character of the energy spectrum of these electrons, the component of the conductivity tensor should be neglected.

Thus, experimental study of magnetoresistance anisotropy and Hall effect at different orientations of current and magnetic field relative to the layers enables us to determine the contribution of various charge carriers groups to the conductivity, as well as to find out the character of FS anisotropy in the plane of layers. 


\title{
References
}

1. Lifshits I.M., Peschansky V.G., Zh. Eksp. Teor. Fiz., 1958, 35, 1251 (in Russian); Sov. Phys. JETP, 1959, 8, 857.

2. Lifshits I.M., Peschansky V.G., Zh. Eksp. Teor. Fiz., 1960, 38, 188 (in Rusian); Sov. Phys. JETP, 1960, 11, 137.

3. von Klizing K., Droda G., Pepper M., Phys. Rev. Lett., 1980, 45, 494.

4. Tsui D.C. , Stromer H.L., Gossard A., Phys. Rev. B, 1983, 28, 2274.

5. Lifshits I.M., Zh. Eksp. Teor. Fiz., 1957, 32, 1509 (in Russian); Sov. Phys. JETP, 1957, 5, 1227.

6. Peschansky V.G., Fiz. Nizk. Temp., 1997, 23, 47 (in Russian); Low Temp. Phys., 1997, 23, 35.

7. Peschansky V.G., Kartsovnik M.V., J. Low Temp. Phys., 1999, 117, 1717.

8. Rousseau R. et al., J. Phys. I (France), 1996, 6, 1527.

9. Kartsovnik M. V. et al., Synth. Metals, 1995, 70, 811.

10. Peschansky V.G., Hasan R.A., Savel'eva S.N., Fizika Metalov i Metalovidenie, 2002, 94, 14 (in Russian).

11. Kartsovnik M. V., Peschansky V.G., Fiz. Nizk. Temp., 2005, 31, 249 (in Russian); Low Temp. Phys., 2005, 31, 185.

12. Singelton J., Rep. Prog. Phys., 2000, 63, 1111-1207.

13. Kartsovnik M.V., Chem. Rev., 2001, 104, 5737.

\section{Єфект Холла у органічних шаруватих провідниках}

\author{
Р.А.Хасан ${ }^{1}$, М.В.Карцовник ${ }^{2}$, В.Г.Піщанський ${ }^{1,3}$ \\ 1 Харківський національний університет ім. В.Н.Каразіна, \\ майд. Свободи 4, Харків, Україна, 1 \\ 2 Інститут Вальтера-Майснера, \\ вул. Вальтера-Майснера 8, \\ 85748 Гарчинг, Німеччина \\ 3 Фізико-технічний інститут \\ низьких температур ім. Б.І Вєркіна, \\ пр. Леніна 47,Харків,Україна, 3
}

Отримано 18 липня 2005 р., в остаточному вигляді 13 лютого 2006 p.

\begin{abstract}
Теоретично розглянуто ефект Холла у органічних шаруватих провідниках з багатолистковою поверхнєю Фермі. Показано, що експериментальне дослідження ефекту Холла та анізотропії магнітоопору при різних орієнтаціях струму та квантуючого магнитного поля відносно шарів дозволяє визначити внесок у провідність різних груп носіїв заряду та встановити характер анізотропії поверхні Фермі у площині шарів.
\end{abstract}

Ключові слова: еФект Холла, органічні шаруваті провідники, багатолисткові поверхні Фермі

PACS: $72.15 . G d, 75.70 . C n$ 